\title{
Initial Erosion Models
}

\author{
A. Young ${ }^{\text {a L.M.A. Tenuta }}{ }^{\mathrm{b}}$

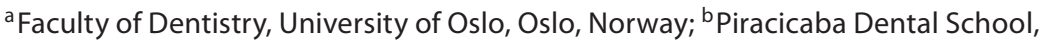 \\ University of Campinas, Piracicaba, Brazil
}

\section{Key Words}

Dentine $\cdot$ Enamel $\cdot$ In situ testing $\cdot$ In vivo models $\cdot$ Saliva Tooth erosion

\begin{abstract}
Initial dental erosion - the very first tooth surface changes caused by short-term acidic exposures before any tissue loss is observed - has been studied using in vitro and in situ/in vivo protocols. This paper describes the events that should be considered when modelling initial erosion, the available protocols and their strengths and limitations. In vitro initial erosion models can provide relevant information about the erosive outcome. However, the published studies vary considerably regarding the erosive parameters used, and few of these studies have been validated. On the other hand, relevant in situ/in vivo studies are available that have studied the effect of salivary pellicle and preventive treatments on initial erosion, as well as the changes in salivary $\mathrm{pH}$ following exposure to acid solutions. Guidelines for good methodology in modelling initial erosion are proposed.
\end{abstract}

Copyright $\odot 2011$ S. Karger AG, Basel

In the process of dental erosion, the initial acid attacks on the tooth surface result in a superficial partial dissolution of mineral - early-stage surface softening [Arends and Ten Cate, 1981] - which can reach a few micrometres into enamel or dentine [Imfeld, 1996; Finke et al., 2000; Addy and Shellis, 2006; Cheng et al., 2009]. If the erosive challenge is more prolonged, the outermost layer of the softened surface will eventually be completely dissolved, resulting in permanent loss of tooth structure (in depth) [Addy and Shellis, 2006; Cheng et al., 2009]. A substantial number of studies have been published on the effects of initial erosion and on methods to reduce or prevent erosion. Unfortunately, the great variety in experimental designs of these studies makes a comparison of the results and a safe extrapolation to the clinical setting difficult. Thus, the aims of this paper are to: (1) discuss important variables to be considered when evaluating initial dental erosion; (2) review currently available in vitro, in situ and in vivo models of initial dental erosion, and (3) propose guidelines for good methodology when modelling this process.

The literature reviewed during the preparation of this paper is available as four online supplementary tables (www.karger.com/doi/10.1159/000325943), which are referred to as online supplementary tables S1-S4 in this paper.

\section{Events during Initial Erosion}

The oral cavity is continuously bathed in saliva, which has a residual volume of approximately $1 \mathrm{ml}$ with a thin salivary film covering the teeth (fig. 1a). When an acidic beverage or fluid enters the mouth, its volume greatly exceeds the amount of saliva present, and for a short period of time (depending on how long the solution is kept in the

\section{KARGER \\ Fax +4161306 1234 \\ E-Mail karger@karger.ch}

www.karger.com (c) $2011 \mathrm{~S}$. Karger AG, Basel

0008-6568/11/0457-0033\$38.00/0

Accessible online at:

www.karger.com/cre
Dr. A. Young

Department of Cariology and Gerodontology

Faculty of Dentistry, University of Oslo

NO-0317 Oslo (Norway)

Tel. +47 22852 190, E-Mail a.y.vik@ odont.uio.no 

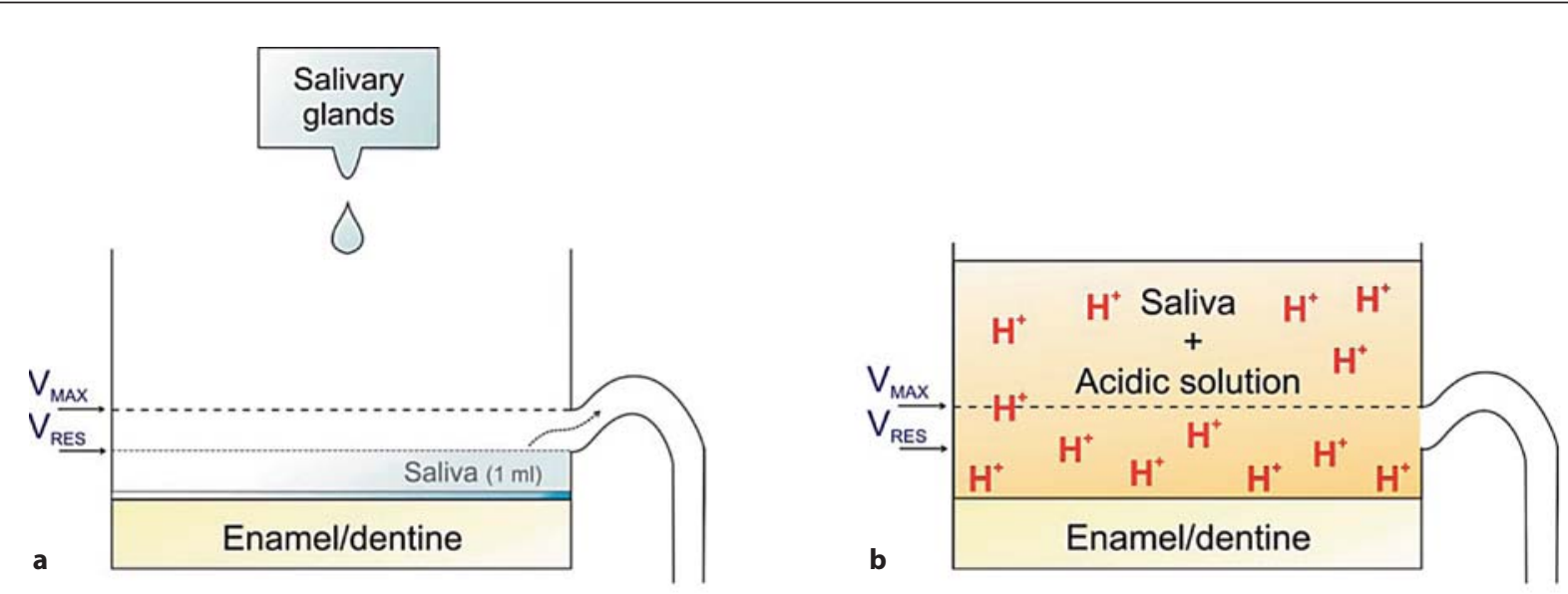

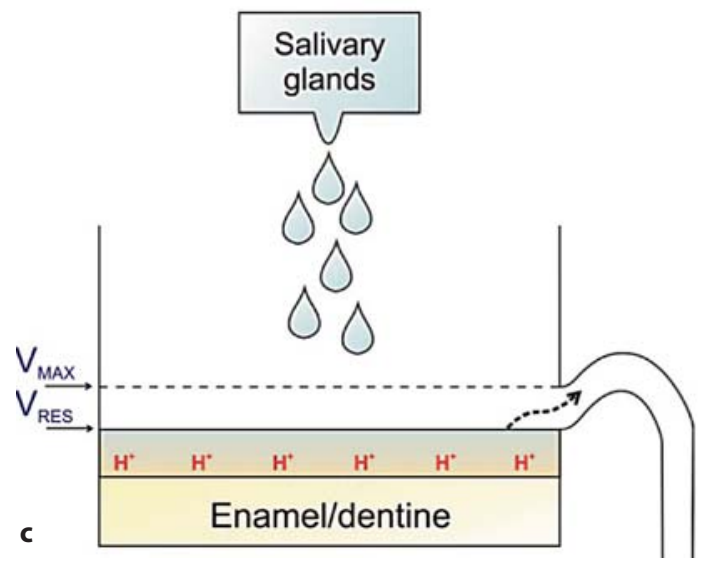

Fig. 1. Events of initial erosion following the entrance of an acidic beverage in the mouth. a-c The siphon represents the removal of liquids from the mouth by swallowing, if $\mathrm{V}_{\mathrm{MAX}}$ (maximum volume of saliva in the mouth before swallowing) is exceeded. $\mathrm{V}_{\mathrm{RES}}=$ Residual volume of saliva in the mouth after swallowing.

mouth) the teeth will be exposed to a mixture of saliva and acid solution that resembles the acid solution itself (fig. 1b). This condition lasts for a while, after which the erosive fluid is either ingested or expectorated. Then, the flux of saliva will immediately start to wash away or buffer the acids (fig. 1c), leaving a partly demineralised surface that is prone to wear or remineralisation [Davis and Winter, 1980; Jaeggi and Lussi, 1999; Attin et al., 2000]. The presence of the acquired pellicle on the tooth surface will partially protect the tooth structure from the erosive attack by acting as a permselective barrier, but this protection is limited and a certain amount of mineral will be lost to the erosive solution [Hara et al., 2006a]. At a closer view of the tooth surface (fig. 1d), the acidic d Enlarged view of the tooth surface showing initial demineralisation. Refer to the text for specific description of each scheme (schematic representation of the oral cavity derived from Ekstrand and Oliveby [1999]). solution will flow over the enamel or dentine, and demineralisation will occur as in an open system; the reaction rate will not slow down because there is little build-up of reaction products.

From this short description, it is clear that many factors can influence the erosive outcome [Lussi, 2006]. Interaction between chemical factors (e.g. the composition of the acidic solution), biological factors (e.g. the presence of saliva/pellicle and salivary flow) and behavioural factors (e.g. the way the acid fluid is kept in the mouth or how teeth are abraded shortly after exposure) plays a significant role, and these factors should be considered when modelling erosion studies. Furthermore, in vivo the erosive challenges will be short and repeated, and inter- 
spersed by events of abrasion and/or remineralisation. Thus, the characteristics of the resulting eroded surface after short acid attacks are important factors to be considered in initial erosion studies.

\section{Important Parameters in Modelling Early Stages of Dental Erosion}

This discussion is restricted to models in which no surface loss in depth is observed but only surface softening. For enamel this can be monitored by, for example, making reference marks, e.g. indentations made by a hardness tester, on the specimen surface before exposure to the erosive challenge in vitro or in situ and checking that the marks are still present after the challenge or, for in vivo models, studying replicas by scanning electron microscopy (SEM).

\section{Tooth Substrate and Specimens}

The substrate usually consists of prepared specimens of permanent or deciduous human or bovine enamel or dentine in in vitro and in situ studies, or natural human tooth enamel surfaces in vivo. Hydroxyapatite powder and/or particles of tooth tissue are unsuitable for studies of initial erosion because checks for surface loss cannot be made. In some in vivo models, natural teeth, such as the upper anterior teeth, were selected for easy accessibility [Young et al., 2006; Sauro et al., 2008; Hjortsjö et al., 2009], while in another study different teeth were included in order to compare varying conditions within the oral cavity [Bartlett et al., 2003].

Most techniques cannot measure initial changes on natural tooth surfaces as they require a planar surface, so tooth specimens must be ground flat first. This process will almost certainly remove approximately $100 \mu \mathrm{m}$, including the aprismatic surface enamel if present [Whittaker, 1982]. It is generally claimed that ground enamel surfaces are more susceptible to acid dissolution [Finke et al., 2000; Ganss et al., 2000; Lussi et al., 2011]. Thus, the requirement of grinding and polishing the surface should be seen as a factor which may affect the response. Sometimes, however, it may be desirable since it eliminates the more variable surface and accelerates the erosive challenge, thereby reducing experimental time. Further research on natural, unpolished enamel surfaces has been recommended to fully understand de- and remineralisation under erosive conditions [Lippert et al., 2004a]. Techniques such as atomic force microscopy and whitelight interferometry, which have been used successfully on native tooth surfaces [Finke et al., 2000; Hove et al., 2008], could help to elucidate the effects of polishing on results.

Bovine incisor enamel and dentine are often used as experimental substitutes for human tissues, which can be difficult to obtain. These substrates are considered acceptable because of their relatively close resemblance to their human counterparts, although bovine tissue has a greater response to acid challenge owing to greater porosity [Arends et al., 1989; Amaechi et al., 1999].

\section{Erosive Challenge}

Erosive Solutions

Numerous epidemiological, clinical and laboratory reports link erosion to excessive consumption of acidic drinks. These drinks are popular mainly because of their refreshing taste, which is due to the presence of various acids with low $\mathrm{p} K_{\mathrm{a}}$ values $\left(\mathrm{p} K_{\mathrm{a} 1}=2.15-4.10\right)$. In fruitbased drinks, citric and malic acids are the most prominent acids. Acidic candies and sprays also commonly contain these acids, and are often consumed over longer periods of time than acidic drinks. Many soft drinks may also contain other acids, such as phosphoric, ascorbic or lactic acids. Wine is also a popular acidic drink containing mainly combinations of tartaric and malic acids, but also smaller amounts of lactic, succinic and citric acids. Carbonic acid, which gives the fizz to many soft drinks, increases the erosive potential of mineral waters by a small, but probably insignificant, amount [Parry et al., 2001].

Therefore, in vitro and in situ studies of initial erosion have used acidic challenges consisting of either plain citric acid, various acidic beverages such as soft drinks (Coca Cola or Sprite: $\mathrm{pH}$ 2.3-3.2), juices (orange, grapefruit, lemon or blackcurrant: $\mathrm{pH} 3-4)$, wines $(\mathrm{pH} 2.9-$ 4.2), acidic candies ( $\mathrm{pH} 2.3-3.1)$ or sprays ( $\mathrm{pH} 1.9-2.3$ ) [Jandt, 2006; Davis et al., 2007; Brand et al., 2009a; Gambon et al., 2009]. Hydrochloric acid has been used in many studies of erosion caused by reflux or by vomiting events often associated with eating disturbances. Although pure gastric fluid has a $\mathrm{pH}$ of $0.9-1.5$, the $\mathrm{pH}$ in the oral cavity is seldom $<1.5$ because of salivary buffering and dilution. In bulimia patients, the vomit has a $\mathrm{pH}$ of 2.9-5.0 [Milosevic et al., 1997]. Hove et al. [2006, 2007, 2008 ] used $0.01 \mathrm{M} \mathrm{HCl}, \mathrm{pH} 2.2$. Attributes of erosive fluids which influence erosive potential are reviewed by Barbour et al. [2011].

\section{Exposure Variables}

Time, volume, flow rate of solutions and consumption habits are probably the main source of variation among 
in vitro and in situ models evaluating initial erosion. To simulate the clinical situation as closely as possible, extensive erosion periods should be avoided, since the resulting eroded surface would no longer retain the same susceptibilities to remineralisation or abrasion [Cheng et al., 2009; Field et al., 2010]. On the other hand, the exposure time and acid challenge have to produce a change measurable by the chosen method. Both factors have to be balanced. In the mouth, the time span the $\mathrm{pH}$ remains low is usually not longer than 2 min [Millward et al., 1997], and similar exposure times should be followed by in vitro and in situ models to better simulate conditions in vivo, unless specific conditions are to be modelled, such as low salivary flow rates or extensive periods of exposure to acids. Some in vitro studies have indeed shown how the prolonged exposure to acids modifies the eroded surface [Cheng et al., 2009], perhaps to an unrealistic condition. Extrapolation of in vitro results is further complicated by variations in stirring rate, which can greatly influence the erosive outcome [Eisenburger and Addy, 2003; Barbour et al., 2005; Shellis et al., 2005; Wiegand et al., 2007]. The rate in in vitro studies can vary from unstirred [Lippert et al., 2004a; Cheng et al., 2009] or mild agitation to continuous agitation at various velocities [Lussi et al., 2000; Lippert et al., 2004b] (online suppl. tables S1, S2). In fact, it is possible that different experimental conditions account for the different conclusions drawn from in vitro studies on the correlation of the erosive challenge with chemical properties.

Some in situ studies have avoided the ethical problem of exposing test subjects to acids by performing the acidic challenge ex vivo. This approach has been commonly used in cyclic challenge studies where the end result is tooth surface loss and not just initial erosion. Initial erosion studies performed completely in situ generally involve short exposure times ranging from a single 15-second exposure and repeated consumption of acidic beverages to a period of up to $1 \mathrm{~h}$ (online suppl. table S3). In many in situ study models, the test exposure is equivalent to a common acidic consumption in the population, and as such can be argued to be ethically unproblematic. Using an ex vivo design for the acidic challenge requires care in maintaining a clinically relevant acidic challenge, while ex vivo salivary pellicle formation is considered inappropriate as a relevant model in in situ studies. In studies on the erosive potential of sucking acidic candies or lollipops, although the exposure time is much greater than that associated with drinking acidic beverages, higher salivary flow rates are reported, at least initially, with candies [Jensdottir et al., 2005; Brand et al., 2009b].
Changes in flow rates will automatically affect salivary clearance rate and the concentrations of buffers.

Professional wine tasters have been included as test subjects in erosion studies as they are at high risk of dental erosion: at work they may test 20-200 different wines daily by rinsing for between $15 \mathrm{~s}$ and several minutes [Brand et al., 2009b]. Brand et al. [2009b] showed that rinsing $5 \mathrm{ml}$ of wine for $15-45 \mathrm{~s}$ caused saliva $\mathrm{pH}$ to fall for several minutes well below the critical $\mathrm{pH}$ for enamel dissolution. For many wines, repetition of this process will render the in vivo effect of saliva relatively ineffective.

\section{Biological Factors}

Acids entering the oral cavity are cleared and neutralised by saliva, so information about the salivary status of test subjects is important. Saliva secretion rates should be measured and reported, and should usually be within normal limits. Where this is not so, it should be stated. For example, in one in situ study the inclusion criterion was hyposalivation [Gedalia et al., 1996], while in another study a single participant was recorded as having reduced salivary secretion [Lussi et al., 1997].

The pellicle and the dental plaque are potentially protective against dental erosion, and the fact that cervical areas of eroded teeth are often unaffected may be attributed to this fact. The pellicle slows down remineralisation overall, although it might favour subsurface remineralisation [Zahradnik, 1979]. Salivary pellicle formed in situ protects enamel from the erosive effects of orange juice to some extent [Hara et al., 2006b; Hannig et al., 2009], even after only $2 \mathrm{~h}$ of formation time [Hannig et al., 2003]. In vitro, pellicle can provide protection against erosion in people consuming acidic beverages hourly or even more often, though this protection was more pronounced for enamel than dentine and varied greatly between individuals [Wetton et al., 2006, 2007]. Several in vitro models have included a period to allow formation of a salivary pellicle before the erosive challenge as a step in the experimental protocol [Hannig et al., 2001, 2003, 2004, 2007; Hove et al., 2007].

The protective effect of the pellicle will largely depend on the strength and duration of the acidic challenge and, when evaluated in vitro or in situ, will be affected by the study design. For example, prolonged immersion in acid solutions may reduce the power of the study to detect a significant effect of the pellicle.

An atomic force microscopy study showed that drinking different acidic drinks can influence the thickness and morphology of pellicle acquired in situ [Finke et al., 
2002], possibly because of differences in ionic strength or thickening agents, or the extent to which they stimulate saliva flow.

\section{Preventive Treatments}

Most treatments and protocols for studying prevention or reduction of erosive tooth loss have been studied under the conditions of initial erosion, such as the use of a topical application on tooth specimens or teeth immediately before an erosive challenge. Single applications of some fluorides have been shown to significantly protect enamel against a one-off acidic challenge [Young et al., 2006; Hjortsjö et al., 2009a] or successive brief acid challenges [Hove et al., 2007; Sauro et al., 2008]. From a study model point of view, on the one hand it is suggested that frequent re-application of a protective treatment is to be avoided in in vitro experimental models, as this may be practically unrealistic for the clinical setting. On the other hand, however, in vitro and in situ testing of the persistence of an effect of preventive measures is necessary and may provide useful information, e.g. during product development, in order to determine appropriate regimes [Hjortsjö et al., 2009b].

\section{In vitro Models}

\section{Erosive Potential of Beverages}

In vitro tests suggest that a number of properties of beverages, such as $\mathrm{pH}$, buffering properties and concentrations of calcium phosphate or fluoride, influence the erosive potential [Barbour et al., 2011]. The physical properties of the solution, such as viscosity and wettability, have also been considered. The results of such studies are likely to be strongly influenced by study design, which varies considerably [Barbour et al., 2011]. In many studies, erosion is assessed by the amount of surface loss from specimens after prolonged exposure to the test beverage [Grenby et al., 1990; Larsen and Nyvad, 1999; Davis et al., 2007; Hara and Zero, 2008; Ehlen et al., 2008; Hunter et al., 2008; Cochrane et al., 2009; Hunter et al., 2009; Murrell et al., 2010]. Under these conditions, mineral loss may be influenced unduly by local factors influencing dissolution, such as the velocity of agitation, or how often an erosive solution is changed, and may not necessarily reflect the erosive potential in a clinical setting. Moreover, after prolonged immersion in erosive solutions, specimen surfaces may no longer maintain the same response to further abrasive or remineralising events, leading to inconclusive or conflicting results. In addition, a closed system, such as a beaker containing an acid solution and a tooth specimen, is different from an open system, such as a solution flowing over tooth surfaces in the mouth and immediately being mixed with saliva. Thus, initial screening tests should not be discussed without considering the influence of the study design. Reliable models taking into consideration the influence of variables, such as stirring, temperature and exposure time, should be preferred.

\section{Initial (Short-Term) in vitro Erosion Models}

It is somewhat difficult to separate models dealing with softening of the surface only and those involving substance loss, because both effects are part of a continuous process [Addy and Shellis, 2006; Cheng et al., 2009]. It is strongly recommended that a check on the absence of substance loss (see above) forms part of the protocol for initial erosion studies. However, given that most papers do not present this verification, in the present paper only protocols concerning exposure to acids for periods up to $10 \mathrm{~min}$ were included, given that at longer times there is loss of tooth structure in depth [Hara and Zero, 2008; Cheng et al., 2009].

Reported initial erosion protocols fall into two categories: those with only an acid exposure and those using cycles of short exposures to acid, followed by exposure to saliva. Online supplementary tables S1 and S2 present a detailed description of some of these protocols available in the literature.

From the analysis of online supplementary tables S1 and S2, it is possible to observe that there is not one widely used in vitro model or protocol; it is in fact difficult to find a paper referring to a specific model chosen by the authors. The relative simplicity of short-term exposure models - immersion in acidic solution and measurement of the resulting early changes - may be the cause of the high number of different research protocols in the literature. The resulting variation in conditions, such as the volume of solution used, the temperature, the flow of solution and time, is correlated with a lack of consistency in results. Therefore, it seems that guidelines are urgently needed if comparable initial erosion models are to be developed. It is possible that models using cycling conditions, with very short exposure to acids and their removal by saliva, better reflect the erosive challenge in the oral cavity. Better yet, open-system models simulating the intermittent flow of the acidic solution and saliva [Attin et al., 2003] could more closely reflect the erosive challenge happening in the mouth. Thus, the use of cycling models should be encouraged. 
Also, inclusion of previous treatment with salivary pellicle is also advisable when the results are to be extrapolated to the clinical situation.

A challenge to research is that none of these models have been validated as surrogates for clinical erosion conditions.

\section{In situ/in vivo Models}

Models using dental specimens in situ (with in situ or ex vivo acidic challenge) and those dealing with the oral cavity and natural teeth as a scenario have been included in the present paper. These studies, outlined in online supplementary tables S3 and S4, can be broadly divided into (a) studies on the protective effect of the enamel pellicle; (b) studies on the detrimental effects of different acidic drinks and sweets, and (c) studies on the effect of different agents for preventing erosive tooth loss. The extent to which these experiments are performed in the human mouth has varied greatly - from formation of pellicles formed in situ in an otherwise in vitro experiment [Hannig et al., 2001] to the entire study being carried out in the oral cavity, including all intra-oral measurements [Bartlett et al., 2003].

\section{Test Subjects}

Involvement of human subjects in any form of research requires approval of the study protocol by a relevant ethical committee, and participants must give their written informed consent prior to participation. The number of test subjects will vary depending upon the aims of the study and the precision of the measurement technique, and must be sufficient to give the study adequate statistical power. Power analyses based on the statistical significance criterion and the magnitude of the effect of interest in the population can be calculated either from pilot studies or from previous study results [Barlow et al., 2009; Hara et al., 2009]. They are now commonly used to decide the number of participants necessary for inclusion, and as a result many ethical committees require early involvement of a statistician in the planning stages of such studies for this purpose.

In the majority of studies (online suppl. tables S3, S4), subjects have been in the age range of 25-50 years, and in several studies it was mentioned that the choice of the subject age group was related to the generally well-known erosive dietary habits of younger adults. It would seem advisable, where possible, to choose test subjects of reasonably similar age, even if the acidic challenge is per- formed outside the mouth. In this way, one can control for other less easily measured intra-oral factors that may affect the erosive effect. For example, in in vivo studies, the longer a tooth has been present in the oral cavity, the more mature the tooth surface enamel becomes, and this maturation process will affect the response of the tooth to acidic challenges [Fejerskov et al., 2008]. With respect to tooth specimens originating from erupted teeth, the age of the tooth donor as well as the fluoride history of the tooth donor should ideally be taken into consideration. It is well known that physiological differences exist in enamel and dentine between subjects, between teeth in the same mouth and between different parts of the same tooth. Although in situ studies using ground and polished tooth specimens will partly compensate for these differences, there is a need for careful standardisation of specimen preparation and randomisation of tooth specimens between subjects used in studies modelling initial dental erosion.

\section{In situ Appliances}

Some in situ studies have employed both maxillary mini-splints and vacuum-formed methacrylate splints [Hannig et al., 2001, 2007], or soft-vacuum-formed splints accommodating intra-oral $\mathrm{pH}$ electrodes [Moazzez et al., 2000; Bartlett et al., 2003]. Depending on the study design, the number of specimens carried by the appliances has varied from 1 to 8 , and these have been fixed by different methods, including taping [Lippert et al., 2004c], specially-made plastic holders using sticky wax and wires [Hara et al., 2006; Zero et al., 2006; Hara et al., 2009; Barlow et al., 2009] and impression material [Hannig et al., 2001,2003, 2007]. When only the initial surface softening changes in the tooth surface are to be studied, the positioning of the samples within the appliance should be such that abrasive and attritive forces are avoided. However, in most published models involving palatal appliances and intra-oral acidic challenges, specimens were directly exposed to sucking, drinking, rinsing or swishing of the acidic agent. In one study, participants were specifically instructed to hold the acidic beverage against the appliance carrying the specimens for $15 \mathrm{~s}$ [Hara et al., 2006b]. In that study, the design of the specimen holder was such that the specimens were protected from mechanical forces. Various designs are used to prevent wear to tooth specimens in initial erosion studies. It is obvious that the positioning of the specimens within one subject must not vary to an extent which will influence the results between specimens. Furthermore, in studies performed over several hours, it should be stated whether 
the appliance is removed while the test subject is eating and performing oral hygiene routines. This aspect is more relevant for models where the appliance is in place over days or weeks and the outcome measurement is tooth surface loss.

\section{Method of Acid Exposure}

In situ models involving specimens mounted in appliances have used either extra-oral or in situ acid challenge. The first type was presumably in most studies designed to avoid unnecessary acid exposure of the teeth of the test subjects. In these studies, the acid was often gently agitated during the exposure, but this was not always specified. In one model, a previous etching functioned as a control against excessive surface loss in the process of selecting specimens [Barlow et al., 2009; Hara et al., 2009]. In the second type of model, a very wide variety of methods has been used to administer the intra-oral acid challenge. For obvious reasons, it is important that the challenge is presented and tested in a manner that is suitable to the aim of the study, and subjects have either sipped, rinsed, drunk or swished beverages, and either actively sucked or passively retained candies in the mouth (online suppl. table S4). A special protocol was used by Brand et al. [2009b] to mimic the routine of professional wine tasters. The subjects rinsed vigorously with different wine samples and for varying lengths of time.

\section{Models Involving Natural Teeth or the Oral Cavity}

\section{SEM and Calcium Analysis}

For ethical and practical reasons, testing of human teeth in vivo has not been very common. A combined in situ replica/SEM technique has been reported [Sauro et al., 2008]. Following a control impression, half of the test teeth were treated with a fluoride agent before subjects drank an acidic beverage. Then a second impression was taken and replicas were compared using SEM. This model provided a visual record of the morphological changes in enamel induced by an acidic drink and the effect of previous treatment with fluoride toothpaste. Although somewhat time-consuming, the model could be used in future studies on initial erosive changes.

Indirect techniques, such as calcium or phosphate analysis, can be used where the acidic challenge is carried out in vivo, but outcome measurements are carried out ex vivo. In a recent in vivo model, the acid challenge was performed in vivo and the calcium content of the acid was determined [Young et al., 2006; Hjortsjö et al., 2009]. The major advantage of this model is that relevant natural tooth substrate with an existing natural salivary pellicle is tested and, providing the acidic challenge is mild and short, then ethical approval can be achieved. Various modifications to this model, such as the isolation of a more precisely defined area of the tooth surface for acidic challenge and treatment, or introduction of a splitmouth technique, could present benefits for improved control of biological variables.

\section{Saliva Analysis}

In some initial dental erosion studies, various salivary parameters, such as secretion rate, $\mathrm{pH}$, calcium and phosphate concentrations, and degree of saturation with respect to hydroxyapatite $\left(\mathrm{DS}_{\mathrm{HAp}}\right)$ have been recorded at baseline and afterwards at various intervals [Jensdottir et al., 2005, 2007]. To avoid loss of $\mathrm{CO}_{2}$, Jensdottir et al. collected saliva through a glass tube into a syringe impermeable to gases. Saliva samples, collected for $5 \mathrm{~min}$ at baseline, every $30 \mathrm{~s}$ during a 4 -min period of stimulation and for 10 min after stimulation, were stored in the syringes on ice, and both $\mathrm{pH}$ and $\mathrm{P}_{\mathrm{CO}_{2}}$ were determined within 30 min using a blood gas analyzer. Using this closed system, they found a good correlation between $\mathrm{DS}_{\mathrm{HAp}}$ measured in situ and the actual dissolution of hydroxyapatite crystals in vitro, indicating that predictive calculations of the erosive potential can also be performed to some extent for saliva. However, the significant role of salivary proteins was theoretically difficult to account for and the erosive potential of solid acidic foodstuff judged from saliva $\mathrm{pH}$ and $\mathrm{DS}_{\mathrm{HAp}}$ may not fully reflect when erosion is likely to occur in the mouth [Jensdottir et al., 2007].

Other in vivo studies have examined changes in salivary flow rate and $\mathrm{pH}$ as a result of intake of acidic candy in the form of lollipops or spray or of different wines [Brand et al., 2009; Gambon et al., 2009]. These studies involved similar study protocols, with saliva collection at baseline, at varying intervals during acid consumption and for some time following acid consumption. Presumably, an open system was used in saliva collection for these studies. Although similar conclusions were drawn from these studies regarding erosive effects of acidic intakes, it would be advisable where possible to collect saliva using a closed system, to prevent loss of $\mathrm{CO}_{2}$ and hence a rise in $\mathrm{pH}$.

Intra-oral $\mathrm{pH}$ has also been examined using close-fitting, vacuum-formed appliances fabricated to hold four miniature antimony electrodes [Moazzez et al., 2000; Bartlett et al., 2003]. The main disadvantage of this method was considered to be the presence of four wires 
Table 1. Initial erosion models in vitro

Models assessing the erosive potential through chemical analysis of acid solutions

- Can be used as screening tools.

- Chemical composition of an acid solution should not be compared with the mineral loss assessed under conditions not simulating clinical conditions, such as prolonged exposure of the tooth substrate to acidic beverages under static conditions. Models controlling for variables affecting mineral dissolution, such as temperature and stirring rate, are recommended for comparison.

\section{Short-term erosion models}

- Validation is urgently needed if models are to be considered representative of the clinical situation. Validation can be made against in situ/in vivo erosive effects, or at least be based on dose responses to predicted outcomes (e.g. greater response to challenges of increasing intensity, such as those generated by acids at increasing concentrations or with increasing erosive effects).

- Time of exposure to acid should be minimal, so that the surface change does not exceed initial erosion but at the same time can be measured accurately.

- Should be carefully designed to avoid the interference of model parameters on the erosive outcome. For example, consistent, reproducible stirring should always be included in the model, and volume/specimen ratios and temperature of solutions should be considered and reported.

- The presence of pellicle is advisable when results are to be extrapolated to the clinical situation.

- Using cycles of exposure to saliva is recommended, since this will more closely resemble the clinical condition.

- Models simulating the flow of erosive solution and saliva on the surface of teeth (as an open system) should be employed.

In situ/in vivo initial erosion studies

- Age and saliva flow for test subjects should be reported.

- Number of tooth specimens/test subjects should be based on power analyses.

- Pellicle should be formed in situ prior to the erosive challenge.

- Validation is needed regarding the expected response to challenges of increasing intensity.

- The acidic challenge, whether this is intra- or extra-oral, must be clinically relevant.

- Factors related to the use of specimens in removable appliances should be relevant for the model (i.e. specimen placement within the appliance or exposure of the specimen to the saliva/acid challenge).

exiting the mouth, especially during longer test periods. The model involved a 20 -min pre-experimental period for the participants to adapt to the splint in order to avoid initial appliance-induced stimulation of salivary flow. It was not practical to place the electrodes on occlusal surfaces because of the risk of damage by opposing teeth during swallowing, and increased retention of acids could be related to the appliance [Bartlett et al., 2003]. An advantage of this model is that subjects functioned as their own controls.

In general, these studies provide relevant information about the role of saliva in the initial stages of dental erosion, and in some cases they can be helpful in the field of product development.

\section{Guidelines for Good Methodology in Initial Erosion Models}

Great variability and lack of validation were found among the studies reviewed. In order to allow comparisons between studies and in order that clinically relevant conclusions can be drawn, guidelines are proposed for initial erosion models (see table 1).

\section{Conclusions}

The very first detrimental effects of acidic solutions on teeth, the changes that occur in the salivary film as well as the effectiveness of preventive treatments for patients can be studied using suitable models. Consideration of the parameters discussed in this paper can assist in the development of standardised study protocols. In this way, results of in vitro and in situ initial erosion studies can be more validly extrapolated to the clinical situation and compared with results from in vivo studies.

\section{Disclosure Statement}

In writing this article, the authors would like to advise that there are no conflicts of interest.

References

\footnotetext{
Addy M, Shellis RP: Interaction between attrition, abrasion and erosion in tooth wear; in Lussi A (ed): Dental Erosion: From Diagnosis to Therapy. Monogr Oral Sci. Basel, Karger, 2006, vol 20, pp 17-31.

-Amaechi BT, Higham SM, Edgar WM: Factors influencing the development of dental erosion in vitro: enamel type, temperature and exposure time. J Oral Rehabil 1999;26:624-630.

-Arends J, Christoffersen J, Ruben J, Jongebloed WL: Remineralization of bovine dentine in vitro. The influence of the F contents in solution on mineral distribution. Caries Res 1989;23:309-314.
} 
Arends J, ten Cate JM: Tooth enamel remineralization. J Crystal Growth 1981;53:135-147.

Attin T, Buchalla W, Gollner M, Hellwig E: Use of variable remineralisation periods to improve the abrasion resistance to previously eroded enamel. Caries Res 2000;34:48-52.

-Attin T, Meyer K, Hellwig E, Buchalla W, Lennon AM: Effect of mineral supplements to citric acid on enamel erosion. Arch Oral Biol 2003;48:753-759.

Barbour ME, Lussi A, Shellis RP: Screening and prediction of erosive potential. Caries Res 2011;45(suppl 1):24-32.

- Barbour ME, Parker DM, Allen GC, Jandt KD: Human enamel erosion in constant composition citric acid solutions as a function of degree of saturation with respect to hydroxyapatite. J Oral Rehabil 2005;32:16-21.

Barlow AP, Sufi F, Mason SC: Evaluation of different fluoridated dentifrice formulations using an in situ erosion remineralization model. J Clin Dent 2009;20:192-198.

- Bartlett DW, Bureau GP, Anggiansah A: Evaluation of the $\mathrm{pH}$ of a new carbonated soft drink beverage: an in vivo investigation. J Prosthodont 2003;12:21-25.

-Brand HS, Gambon DL, Paap A, Bulthuis MS, Veerman ECI, Nieuw Amerongen AV: The erosive potential of lollipops. Int Dent J 2009a;59:358-362.

Brand HS, Tjoe Fat GM, Veerman ECI: The effects of saliva on the erosive potential of three different wines. Aust Dent J 2009b;54: 228-232.

Cheng Z-J, Wang X-M, Cui F-Z, Ge F, Yan J-X: The enamel softening and loss during early erosion studied by AFM, SEM and nanoindentation. Biomed Mater 2009;4:1-7.

Cochrane NJ, Cai F, Yuan Y, Reynolds EC: Erosive potential of beverages sold in Australian schools. Aust Dent J 2009;54:238-244.

-Davis RE, Marshall TA, Qian F, Warren JJ, Wefel JS: In vitro protection against dental erosion afforded by commercially available, calcium-fortified 100 percent juices. J Am Dent Assoc 2007;138:1593-1598.

Davis WB, Winter PJ: The effect of abrasion on enamel and dentine after exposure to dietary acids. Br Dent J 1980;148:253-256.

-Ehlen LA, Marshall TA, Qian F, Wefel JS, Warren JJ: Acidic beverages increase the risk of in vitro tooth erosion. Nutr Res 2008;28: 299-303.

-Eisenburger M, Addy M: Influence of liquid temperature and flow rate on enamel erosion and surface softening. J Oral Rehabil 2003; 30:1076-1080.

Ekstrand J, Oliveby A: Fluoride in the oral environment. Acta Odontol Scand 1999;57:330333.

Fejerskov O, Nyvad B, Kidd EAM: Pathology of dental caries; in Fejerskov O, Kidd EAM (eds): Dental Caries. The Disease and Its Clinical Management. Oxford, Blackwell Munksgaard, 2008, p 21.
Field J, Waterhouse P, German M: Quantifying and qualifying surface changes on dental hard tissues in vitro. J Dent 2010;38:182-190.

Finke M, Jandt KD, Parker DM: The early stages of native enamel dissolution studied with atomic force microscopy. J Colloid Interface Sci 2000;232:156-164.

Finke M, Parker DM, Jandt KD: Influence of soft drinks on the thickness and morphology of in situ acquired pellicle layer on enamel. J Colloid Interface Sci 2002;251:263-270.

Gambon DL, Brand HS, Nieuw Amerongen AV: The erosive potential of candy sprays. $\mathrm{Br}$ Dent J 2009;206:1-4.

-Ganss C, Klimek J, Schwarz N: A comparative profilometric in vitro study of the susceptibility of polished and natural human enamel and dentine surfaces to erosive demineralization. Arch Oral Biol 2000;45: 897-902.

Gedalia I, Braunstein E, Lewinstein I, Shapira L, Ever-Hadani P, Sela MO: Fluoride and hard cheese exposure on etched enamel in neckirradiated patients in situ. J Dent 1996;24: 365-368.

Grenby TH, Mistry M, Desai T: Potential dental effects of infants' fruit drinks studied in vitro. Br J Nutr 1990;64:273-283.

Hannig C, Becker K, Häusler N, Hoth-Hannig W, Attin T, Hannig M: Protective effect of the in situ pellicle on dentin erosion - an ex vivo pilot study. Arch Oral Biol 2007;52:444449.

Hannig C, Berndt D, Hoth-Hannig W, Hannig M: The effect of acidic beverages on the ultrastructure of the acquired enamel pellicle - an in situ study. Arch Oral Biol 2009;54: 518-526.

Hannig M, Balz M: Protective properties of salivary pellicles from two different intraoral sites on enamel erosion. Caries Res 2001;35: 142-148.

Hannig M, Fiebiger M, Güntzer M, Döbert A, Zimehl R, Nekrashevych Y: Protective effect of the in situ-formed short-term salivary pellicle. Arch Oral Biol 2004;49:903-910.

-Hannig M, Hess NJ, Hoth-Hannig W, de Vrese $\mathrm{M}$ : Influence of salivary pellicle formation time on enamel demineralization - an in situ pilot study. Clin Oral Investig 2003; 7:158161.

- Hara AT, Ando M, González-Cabezas C, Cury JA, Serra MC, Zero DT: Protective effect of pellicle against erosive challenges in situ. J Dent Res 2006b;85:612-616.

- Hara AT, Kelly SA, González-Cabezas C, Eckert GJ, Barlow AP, Mason SC, Zero DT: Influence of fluoride availability of dentifrices on eroded enamel remineralization in situ. Caries Res 2009;43:57-63.

Hara AT, Lussi A, Zero DT: Biological factors; in Lussi A (ed): Dental Erosion: From Diagnosis to Therapy. Monogr Oral Sci. Basel, Karger, 2006, vol 20, pp 88-99.

-Hara AT, Zero DT: Analysis of the erosive potential of calcium-containing acidic beverages. Eur J Oral Sci 2008;116:60-65.
Hjortsjö C, Jonski G, Thrane PS, Saxegaard E, Young A: The effects of acidic fluoride solutions on early enamel erosion in vivo. Caries Res 2009a;43:126-131.

Hjortsjö C, Jonski G, Thrane PS, Saxegaard E, Young A: Effect of stannous fluoride and dilute hydrochloric acid on early enamel erosion over time in vivo. Caries Res 2009b;43: 449-454.

- Hove LH, Holme B, Øgaard B, Willumsen T, Tveit AB: The protective effect of $\mathrm{TiF}_{4}, \mathrm{SnF}_{2}$ and $\mathrm{NaF}$ on erosion of enamel by hydrochloric acid in vitro measured by white light interferometry. Caries Res 2006;40:440-443.

Hove LH, Holme B, Young A, Tveit AB: The erosion-inhibiting effect of $\mathrm{TiF}_{4}, \mathrm{SnF}_{2}$, and $\mathrm{NaF}$ solutions on pellicle-covered enamel in vitro. Acta Odontol Scand 2007a;65:259-264.

Hove LH, Holme B, Young A, Tveit AB: The protective effect of $\mathrm{TiF}_{4}, \mathrm{SnF}_{2}$ and $\mathrm{NaF}$ against erosion-like lesions in situ. Caries Res 2008; 42:68-72.

Hove LH, Young A, Tveit AB: An in vitro study on the effect of $\mathrm{TiF}_{4}$ treatment against erosion by hydrochloric acid on pellicle-covered enamel. Caries Res 2007b;41:80-84.

- Hunter M, Patel R, Loyn T, Morgan MZ, Fairchild R, Rees JS: The effect of dilution on the in vitro erosive potential of a range of dilutable fruit drinks. Int J Paediatr Dent 2008; 18:251-255.

Hunter L, Patel S, Rees J: The in vitro erosive potential of a range of baby drinks. Int J Paediatr Dent 2009; 19:325-329.

Imfeld T: Dental erosion. Definition, classification and links. Eur J Oral Sci 1996;104(2 Pt2):151-155.

Jaeggi T, Lussi A: Toothbrush abrasion of erosively altered enamel after intraoral exposure to saliva: an in situ study. Caries Res 1999;33:455-461.

-Jandt KD: Probing the future in functional soft drinks on the nanometer scale - towards tooth friendly drinks. Trends Food Sci Technol 2006; 17:263-271.

-Jensdottir T, Nauntofte B, Buchwald C, Bardow A: Effects of sucking acidic candy on wholemouth saliva composition. Caries Res 2005; 39:468-474.

-Jensdottir T, Nauntofte B, Buchwald C, Bardow A: Effects of calcium on the erosive potential of acidic candies in saliva. Caries Res 2007; 41:68-74.

Larsen JM, Nyvad B: Enamel erosion by some soft drinks and orange juices relative to their $\mathrm{pH}$, buffering effect and contents of calcium phosphate. Caries Res 1999;33:81-87.

Lippert F, Parker DM, Jandt KD: Susceptibility of deciduous and permanent enamel to dietary acid-induced erosion studied with atomic force microscopy nanoindentation. Eur J Oral Sci 2004a;112:61-66.

Lippert F, Parker DM, Jandt KD: In vitro demineralization/remineralization cycles at human tooth enamel surfaces investigated by AFM and nanoindentation. J Colloid Interface Sci 2004b;280:442-448. 
Lippert F, Parker DM, Jandt KD: In situ remineralisation of surface softened human enamel studied with AFM nanoindentation. Surface Sci 2004c;553:105-114.

Lussi A: Erosive tooth wear - a multifactorial condition of growing concern and increasing knowledge; in Lussi A (ed): Dental Erosion: From Diagnosis to Therapy. Monogr Oral Sci. Basel, Karger, 2006, vol 20, pp 1-8.

Lussi A, Kohler N, Zero D, Schaffner M, Megert B: A comparison of the erosive potential of different beverages in primary and permanent teeth using an in vitro model. Eur J Oral Sci 2000;108:110-114.

-Lussi A, Portmann P, Burhop B: Erosion on abraded dental tissues by acid lozenges: an in situ study. Clin Oral Investig 1997;1:191194.

Lussi A, Schlueter N, Rakhmatullina E, Ganss C: Dental erosion - an overview with emphasis on chemical and histopathological aspects. Caries Res 2011;45(suppl 1):2-12.

-Millward A, Shaw L, Harrington E, Smith AJ: Continuous monitoring of salivary flow rate and $\mathrm{pH}$ at the surface of the dentition following consumption of acidic beverages. Caries Res 1997;31:44-49.
Milosevic A, Brodie DA, Slade PD: Dental erosion, oral hygiene and nutrition in eating disorders. Int J Eat Disord 1997;21:195-199.

Moazzez R, Smith BGN, Bartlett DW: Oral pH and drinking habit during ingestion of a carbonated drink in a group of adolescents with dental erosion. J Dent 2000;28:395-397.

Murrell S, Marshall TA, Moynihan PJ, Qian F, Wefel JS: Comparison of in vitro erosion potentials between beverages available in the United Kingdom and the United States. J Dent 2010;38:284-289.

- Parry J, Shaw L, Arnaud MJ, Smith AJ: Investigation of mineral waters and soft drinks in relation to dental erosion. J Oral Rehabil 2001; 28:766-772.

Sauro S, Mannocci F, Piemontese M, Mongiorgi R: In situ enamel morphology evaluation af ter acidic soft drink consumption: protection factor of contemporary toothpaste. Int J Dent Hyg 2008;9:188-192.

-Shellis RP, Finke M, Eisenburger M, Parker DM, Addy M: Relationship between enamel erosion and liquid flow rate. Eur J Oral Sci 2005; 113:232-238.
Wetton S, Hughes J, Newcombe RG, Addy M: The effect of saliva derived from different individuals on the erosion of enamel and dentine. A study in vitro. Caries Res 2007;41: 423-426.

Wetton S, Hughes J, West NX, Addy M: Exposure time of enamel and dentine to saliva for protection against erosion. A study in vitro. Caries Res 2006;40:213-217.

Whittaker DK: Structural variations in the surface zone of human tooth enamel observed by scanning electron microscopy. Arch Oral Biol 1982;27:383-392.

Wiegand A, Stock A, Attin R, Werner C, Attin T: Impact of the acid flow rate on dentin erosion. J Dent 2007;35:21-27.

- Young A, Thrane PS, Saxegaard E, Jonski G, Rölla G: Effect of stannous fluoride toothpaste on erosion-like lesions: an in vivo study. Eur J Oral Sci 2006;114:180-183.

Zahradnik RT: Modification by salivary pellicles of in vitro enamel remineralization. J Dent Res 1979;58:2066-2073.

Zero DT, Hara AT, Kelly SA, González-Cabezas C, Eckert GJ, Barlow AP, Mason SC: Evaluation of a desensitizing test dentifrice using an in situ erosion remineralization model. J Clin Dent 2006;17:112-116. 